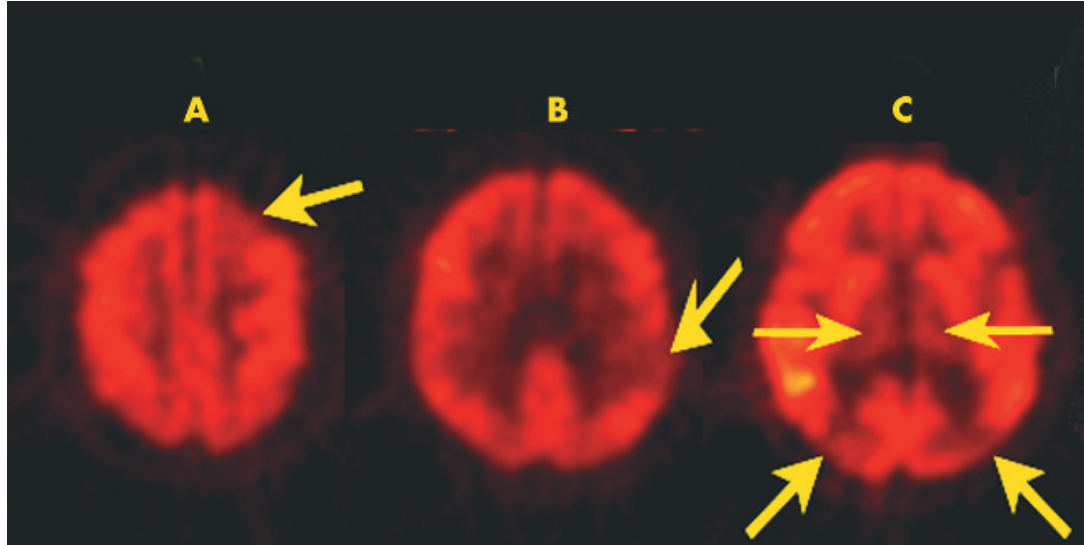

FDG PET

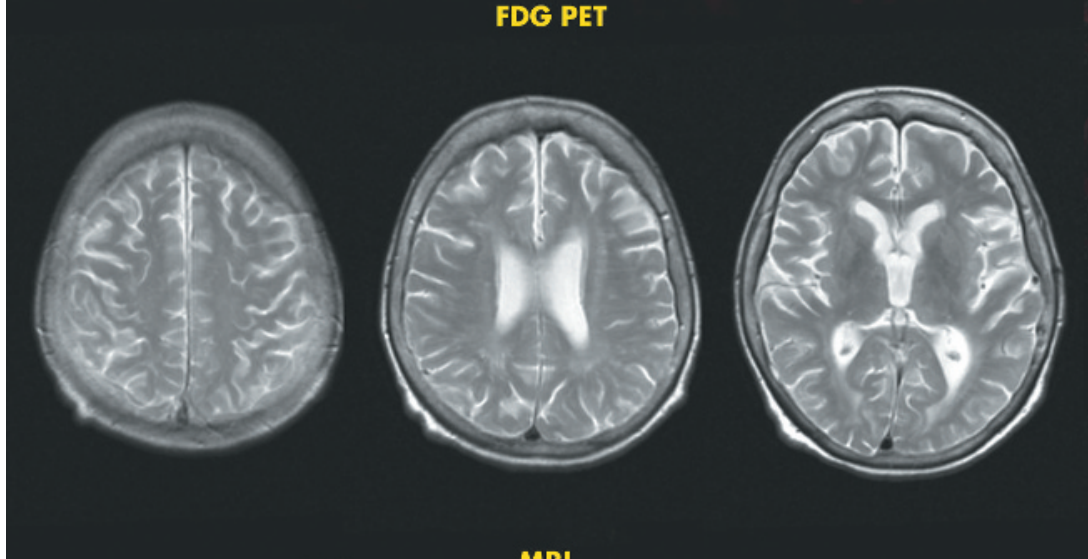

MRI

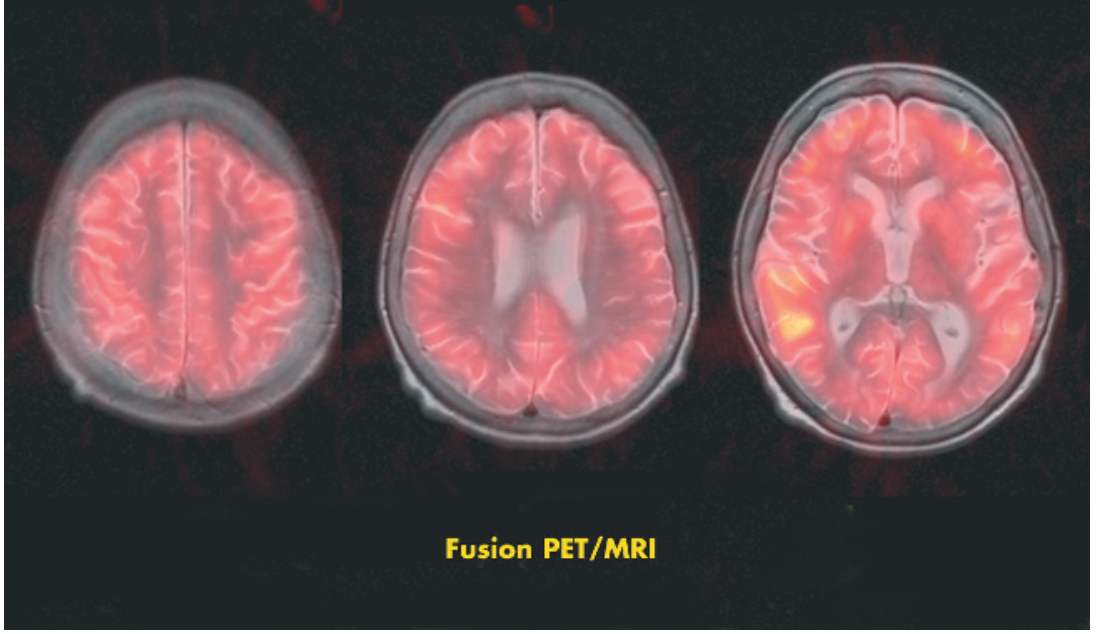

Figure 1 Image fusion of transversal slices each of positron emission tomography (PET) and T2weighted magnetic resonance imaging (MRI). Decreased uptake of ${ }^{18} \mathrm{~F}$ fluorodeoxy-glucose $\left({ }^{18} \mathrm{~F}\right.$ FDG; upper row) in the left frontal cortex (A, arrow), left parietal cortex ( $B$, arrow), left and right parietooccipital cortex (C, thick arrows) and both thalami (C, thin arrows)

biochemical assessment in such patients to avoid missing these sorts of diagnosis.

\section{G Trendelenburg}

Department of Neurology, Charité, Campus Mitte Humboldt University, Berlin, Germany

M T Vanier Fondation Gillet-Mérieux Laboratory, Lyon-Sud University Hospital, Pierre-Bénite, France

S Maza

Clinic for Nuclear Medicine, Charité, Humbold University, Berlin, Germany
G Millat

Fondation Gillet-Mérieux Laboratory, Lyon-Sud University Hospital, Pierre-Bénite, France

G Bohner

Department of Radiology, Neuroradiology Section, Charité, Berlin

D L Munz

Clinic for Nuclear Medicine, Charité, Humbold University, Berlin, Germany

R Zschenderlein

Department of Neurology, Charité Campus Mitte, Humboldt University, Berlin, Germany
Correspondence to: Dr George Trendelenburg, Department of Neurology, Charité Campus Mitte, Humboldt University, Schumannstr 20/21, D-10098 Berlin, Germany; george.trendelenburg@charite.de

doi: 10.1136/innp.2005.086785

\section{References}

1 Vanier MT, Millat G. Niemann-Pick disease type C. Clin Genet 2003;64:269-81

2 Patterson MC. A riddle wrapped in a mystery understanding Niemann-Pick disease, type $\mathrm{C}$. Neurologist 2003;9:301-10.

3 Klünemann HH, lbach B, Guerlach G, et al. Neurologic, psychiatric and neuropsychologic evaluation combined with F-18-fluorodeoxyglucose (FDG)-PET neuroimaging in adult patients with Niemann-Pick disease type C. Ann Neurol 2001;50:S61

4 Lachmann RH, te Vruchte D, Lloyd-Evans E, et al. Treatment with miglustat reverses the lipidtrafficking defect in Niemann-Pick disease type C. Neurobiol Dis 2004; 16:654-8.

5 Cox TM. Substrate reduction therapy for lysosomal storage diseases. Acta Paediatr Suppl 2005;94:69-75.

\section{Late recovery from permanent traumatic vegetative state heralded by event-related potentials}

A post-traumatic vegetative state that holds for more than 12 months is defined as "permanent", implying a very low probability of regaining consciousness. Here we report on a patient who emerged from a traumatic vegetative state after 20 months. Beginning from the 6th month, we observed an improvement in the event-related potentials (ERPs) of his brain to complex sensory and verbal stimulation, although the clinical examinations remained unchanged. The possible role of ERPs as predictors of regaining consciousness after a vegetative state is discussed.

The vegetative state is a most severe neurological syndrome and includes the loss of all kinds of conscious behaviour despite preserved wakefulness. ${ }^{\prime}$ In patients with traumatic head injury, a vegetative state that holds for more than 1 year is considered to be "permanent", which implies irreversibility. In fact, even minimal improvement after this period is extremely improbable. From time to time, however, cases of late emergence are reported, ${ }^{23}$ sometimes even 5 years after the incident. ${ }^{4}$ The statement "The available data are insufficient to provide a trustworthy estimate of the incidence of late improvement" (Childs and Merger, ${ }^{3}$ p 24) is still valid; figures varying as broadly as from $1.6 \%$ to $14 \%$ have been reported. ${ }^{1}$

Given the rarity of such cases, the slightest hint that such an unexpected improvement may occur would be useful. Here we describe a patient in whom cognitive components of cortical ERPs were consistently obtained for more than 1 year before clinical recovery.

A 28-year-old man was admitted to an intensive care unit with a Glasgow Coma Scale score of 3 after a severe car accident. After 5 days, he regained vigilance but had diminished gaze fixation and could not follow even the simplest commands. Intensive stimulation resulted in generalised flexor responses. Three months after the incident, he was transferred to the rehabilitation hospital with a Disability Rating Scale score of 24 (ie, 29, most severe vegetative 
state; 0 , no disability), which remained at that level for the next 17 months. The diagnosis was persistent vegetative state. Multiple cortical lesions were seen on MRI, mainly in the temporal lobe and, to a smaller extent, in the parietal and occipital lobes, with the white matter being damaged to a larger extent than the grey matter. An electroencephalogram showed diffuse moderate slowing with the dominant rhythm of 5-6 Hz, unresponsive to light. Somatosensory and visual evoked potentials were delayed, but brainstem auditory evoked potentials were normal

To evaluate the patient's cognitive abilities independently of his behavioural responses, we used a battery of cognitive auditory ERP tasks. Oddball tasks included the presentation of two non-verbal stimuli (eg, tones), one of which was frequent $(85 \%)$ and the other rare $(15 \%)$. In the semantic oddball task, the rare and frequent stimuli were replaced by rare (Hebrew common words) and frequent (pronounceable non-words) stimulus categories. Each item had two syllables and three to five letters. Semantic congruence tasks included (a) pairs of strongly associated one-syllable words (eg, day-night) as against unrelated words (eg, fish-hand), the associative strength being assessed by independent raters; and (b) simple sentences with a highly expected ending word as against sentences with an incongruent ending word. As this stimulation requires maintaining a sentence in working memory, it can be too demanding for patients with severe disabilities. Fortunately, Hebrew permits the use of short sentences, each containing only three one-syllable words, thus decreasing memory load. During presentation, the ERPs were recorded from seven scalp positions referenced to linked mastoids connected through a $15 \mathrm{k} \Omega$ shunt. The impedances were kept below $7 \mathrm{k} \Omega$. Ocular artefacts were corrected using a regression procedure. The methods of stimulus presentation, data acquisition and analysis have been described in detail previously. ${ }^{5}$ Informed consent to conduct the study was obtained from the patient's parents.
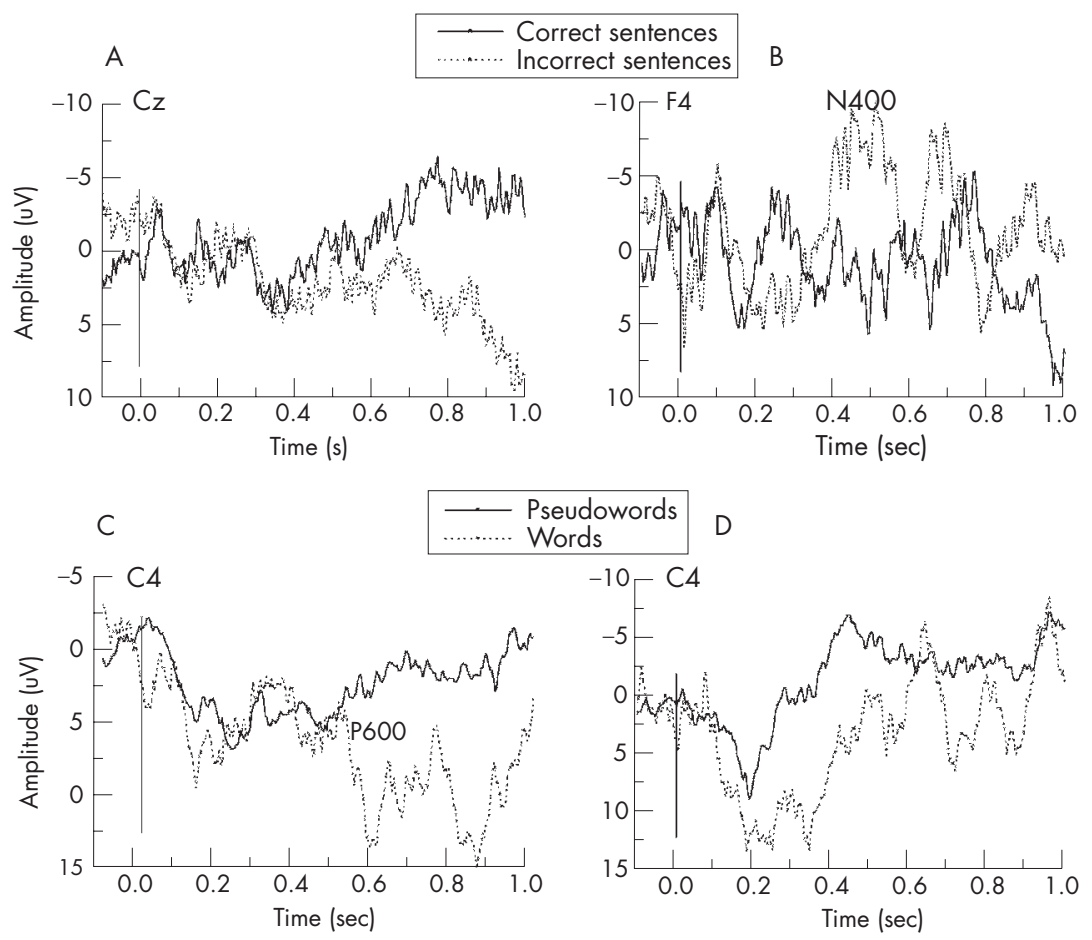

Figure 1 Results of two event-related potential experiments: sentences with semantically correct (solid line) and incorrect (dashed line) final words (A and B), and the semantic oddball with words (dashed line) and pseudowords (solid line; $C$ and D). The left panels ( $A$ and $C$ ) show the results of the fourth examination conducted 6 months after the incident, and the right panels (B and D), the fifth examination 6 months later. In healthy people, incorrect sentence endings usually elicit a negativity N400, often followed by a late positive wave. The patient's dataset $A$ shows a late positive shift in incorrect trials, which alone does not occur in controls. The meaning of such statistically significant but pathological responses remains unclear; hypotheses about possible similarities with brain responses in sleep remain speculative. In (B), incorrect items elicit a typical N400, which remained in the following examinations as well. The response in the semantic oddball (C and D) presents a late positivity (P3) over the posterior cortex, also characteristic for controls. The relatively long P3 latency in $(C)$ is not surprising, as this wave is often delayed even in mild brain injury. The reliability of the observed between-condition differences (ie, correct $v$ incorrect sentences, or words $v$ pseudowords) was evaluated with a running $t$ test and required a certain minimum number $M$ of consecutive significant $t$ values, where $M$ is a function of the length of the interval in which a response can reasonably be looked for, the number of trials and the autocorrelation of the electroencephalogram that was assumed to be $0.9 .^{5}$ According to this criterion, the patient's responses in (A-C) were appreciable, but the P3 response in (D), despite its similarity to normal responses, was not. After recovery, responses in the oddball tasks and in both semantic incongruence tasks were notable.

Electrophysiological examinations were conducted 3, 4, 6, 12 and 18 months after the incident, as well as after recovery (28 months); on each occasion the patient was free of sedative drugs. Despite the intact brainstem auditory evoked potentials, no cortical ERPs were elicited during the two initial examinations. The third examination, however, showed a clear P300 in an oddball task as well as a differentiated response in the word-pair task indicating cortical processing of word meaning. The following two examinations (fig 1) showed relevant or close-to-relevant responses in two of the most difficult conditions: semantic oddball and incongruent sentences. Clinical and neuropsychological (Disability Rating Scale) examinations showed no behavioural improvement that time, resulting in a clinicalphysiological discordance. Only about 20 months after the incident, after intensive physical and speech therapy, the patient suddenly began to articulate distinguishable sounds and then words and, finally, to answer questions about his personal life and the events preceding the crash. Two months later, he was completely aware of his own situation, recognised his family and friends, and could pray. His Disability Rating Scale score at that time was 17. Although the patient could correctly recollect his previous life, he could not consolidate new information, which resulted in the diagnosis of anterograde amnesia.

This instance shows that a patient with traumatic brain injury can regain consciousness even after 20 months in a vegetative state. This clinical improvement was preceded, for more than l year, by an improvement in neurophysiological (cortical ERP) data, indicating the ability of the patient's brain to differentiate both physical and semantic stimulus features, notwithstanding the clinical and neuropsychological pattern of the vegetative state.

We may assume that 6 months after the injury, ERPs already reflected the patient's ability to consciously perceive at least a portion of the presented stimuli. Thus, the patient was most probably in a minimally conscious state all that time despite the consistent negative results of repeated clinical examinations. This supposition implies that ERP findings are superior to clinical data as indices of conscious awareness, a conclusion not warranted by the ERP literature. ${ }^{7} \mathrm{~A}$ more cauticus explanation is that the electrophysiological findings showed cortical processing of stimuli that the patient was not aware of. The ERPs, therefore, manifested processes below clinical significance, which, however, announced the brain's potential to recover.

More data are needed to evaluate the role of ERPs as possible predictors of late recuperation. Somatosensory evoked potentials are particularly useful as predictors of poor outcome in coma. ${ }^{8}$ Another ERP component, the Mismatch Negativity, is a reliable predictor of good outcome in acute coma ${ }^{8}$ and, probably, in vegetative state. ${ }^{9}$ Those data, however, concern patients who recovered within several days to 10 months after the incident, and the predictive values cannot be generalised onto permanent vegetative state. Nevertheless, this case indicates the importance of repetitive neurophysiological (ERP, functional imaging) evaluations of patients in vegetative state who may show fluctuations in cognitive capabilities because of periodical alternations in vigilance. 


\section{Acknowledgements}

This study was supported by the German Federal Ministry of Education and Research (BMBF) and by the German Research Society (DFG) as a part of the SFB 550. We thank T Hinterberger, L Sazbon and Z Groswasser.

S Faran

Institute of Medical Psychology and Behaviora Neurobiology, University of Tubingen, Tubingen,

Germany

J J Vatine, A Lazary Reuth Medical Center, Tel Aviv, Israel

A Ohry

Department of Rehabilitation, Sackler School of Medicine, Tel Aviv University, Tel Aviv, Israe

N Birbaumer Institute of Medical Psychology and Behavioral Neurobiology

B Kotchoubey

Institute of Medical Psychology and Behavioral

Neurobiology

Correspondence to: Dr Boris Kotchoubey, Institute of Medical Psychology and Behavioral Neurobiology University of Tubingen, Gartenstrasse 29, D-72074 Tubingen, Germany; boris.kotchoubey@ uni-tuebingen.de
Ethical approval: The ethical committee of the University of Tubingen, Faculty of Psychology approved the study. Informed consent was obtained from the patient's parents.

doi: 10.1136/jnnp.2005.076554

Competing interests: None declared.

\section{References}

1 Jennett B. The vegetative state. Cambridge, UK University Press, 2002

2 Andrews K. Recovery of patients after four months or more in the persistent vegetative state. BMJ 1993;306:1597-600

3 Childs NL, Merger WN. Brief report: late improvement in consciousness after posttraumatic vegetative state. $N$ Engl J Med $1996 ; 334: 24-5$

4 Dyer C. Hillsborough survivor emerges from permanent vegetative state. BMJ 1997;314:993.

5 Hinterberger T, Wilhelm B, Mellinger J, et al. A tool for detection of cognitive brain functions in severely brain injured patients integrated in the thought-translation-device. IEEE Trans Biomed Eng 2005;52:211-20.

6 Neumann N, Kotchoubey B. Assessment of cognitive functions in severely paralysed and severely brain-damaged patients: neuropsychological and electrophysiological techniques. Brain Res Prot 2004;14:25-36.

7 Kotchoubey B. Event-related potential markers of conscious awareness: two equations with three unknown. In: Laureys S, ed. The boundaries of consciousness: neurobiology and neuropathology. Progr Brain Res, $2005 ; 150: 431-48$

8 Fischer $\mathrm{C}$, Luaute J, Adeleine $\mathrm{P}$, et al. Predictive value of sensory and cognitive evoked potentials for awakening from coma. Neurology 2004;63:669-73.

9 Kotchoubey B, Lang S, Mezger G, et al. Information processing in severe disorders of consciousness: vegetative state and minimally conscious state. Clin Neurophysiol $2005 ; 116: 2441-53$

\section{CORRECTION}

doi: 10.1136/jnnp.2004.050666corr 1

Several corrections were not included in the following paper before it was published in the July issue: M Vaugoyeau, $F$ Viallet, $R$ Aurenty, et al. Axial rotation in Parkinson's Disease (J Neurol Neurosurg Psychiatry 2006; 77:815-21). We apologise for this error. The corrected paper can be viewed online at http://jnnp.com/cgi/reprint/77/7/815.pdf 